\title{
Convolutional and Spiking Neural Network Models for Crop Yield Forecasting
}

\author{
${ }^{*}$ Dr.G.Karuna ${ }^{1}$, K.Pravallika ${ }^{2}$, Dr. K. Anuradha ${ }^{3}$, V. Srilakshmi ${ }^{4}$ \\ ${ }^{1}$ Professor, Computer Science and Engineering, GRIET, Hyderabad, Telangana, India. \\ ${ }^{2}$ PG Scholar, Computer Science and Engineering, GRIET, Hyderabad, Telangana, India. \\ ${ }^{3}$ Professor, Computer Science and Engineering, GRIET, Hyderabad, Telangana, India. \\ ${ }^{4}$ Assistant Professor, Computer Science and Engineering, GRIET, Hyderabad, Telangana, India.
}

\begin{abstract}
Prediction of Crop yield focuses primarily on agriculture research which will have a significant effect on making decisions such as import-export, pricing and distribution of specific crops. Predicting accurately with well-timed forecasts is important, but it is a difficult task due to numerous complex factors. Mostly crops like wheat, rice, peas, pulses, sugar cane, tea, cotton, green houses, corn, and soybean can all be used to forecast crop yields. We considered corn dataset to predict the yield for 13 different states in United States. Crop development and progression are strongly affected by climatic changes and unpredictability. Predicting crop yield well before harvest time will support farmers for selling and storing their crops. Agriculture involves large datasets and knowledge processes. Factors such as Weather Components, Soil Components, Management practices, genotype and their interactions are used in predicting Corn Yield. Precise crop growth generally necessitates a complete overview of the functional correlations between yield and all these interactive variables, which necessitates the use of large datasets and complex algorithms to demonstrate. Various Machine Learning models, Deep Learning models, and Artificial Neural Network algorithms are used for predicting. Deep Neural Network Models such as Convolution Neural Networks (CNN), Spiking Neural Networks (SNN), and Recurrent Neural Networks (RNN) are used to assess corn yield. Integrating CNN, RNN and SNN models outperformed than individual model performance.
\end{abstract}

Keywords - crop yield, spiking neural networks, prediction, recurrent neural networks.

\section{Introduction}

Crop yield forecasting plays a vital role for global food Production. To improve food and nutrition security, decision makers can use efficient process to make timely import and export choices. In order to breed better varieties for different types of environments, seed companies must forecast new hybrids. With the help of crop predictions farmers can be benefited to avoid the losses financially and can be known before what crop should be grown in which season and what are the precautions need to be taken according to the environment and soil interactions.

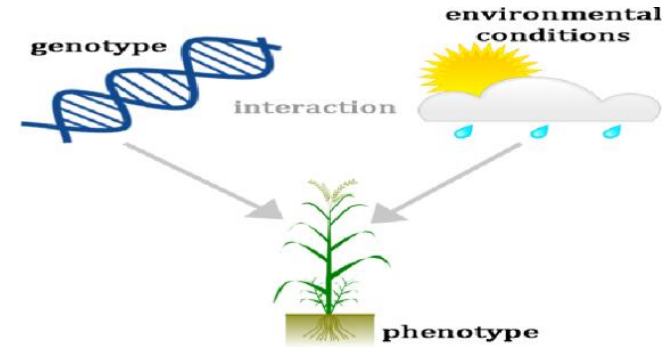

Fig 1.Genotype, phenotype and environmental interactions

Seed companies as well as farmers are going to get benefited ultimately with the help of forecasting. Crop yield forecasting can be done based on the respective factors like soil components, weather components, location, county, latitude and longitude, genotype and

\footnotetext{
*Corresponding author: karunavenkatg@gmail.com
} 
phenotype characteristics etc. It should be known before that how much area should be taken to plant the crop and the plantation week should be noted up to how many months the crop should last all the measures needs to be analyzed before while planting the crops. What crops need to be grow and what crops cannot grow need to be considered based on the past years experiences and can also gather information from the companies like Syngenta[2] which is a global supplier of crop protection products like (herbicides, fungicides, and insecticides), seeds like (Rice and Corn) and other related products. Interactions between genotype and environment [1] are extremely complex. The term genotype refers to an individual's genetic characteristics for a specific trait, whereas phenotype refers to observable characteristics such as physical, physiological, biochemical, and behavioral characteristics. Plant length, for example, is a type of phenotype trait as shown in above Figl and below Fig 2

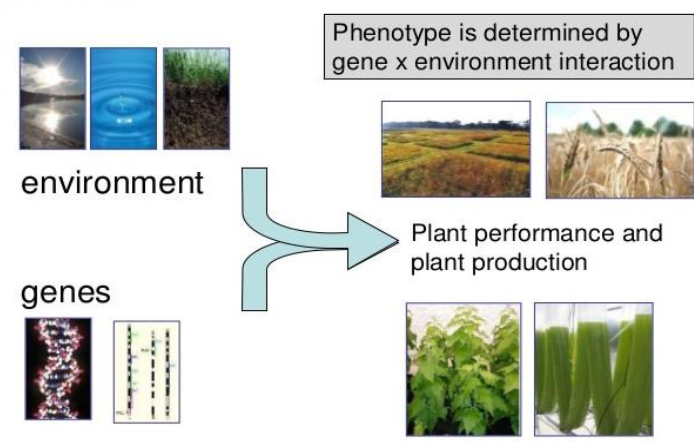

Fig 2.Phenotype traits, Genes and Environment

Millions of markers are usually present in high dimensional marker data, which is defined by genotype for every plant individual. It is important to compute the impact of genetic markers, which usually result in interactions with various environmental influences as well as field management activities.

Alleles are another word for genes, and each individual inherits two alleles for each character, one from male and one from female. Two alleles can be the same (TT or $\mathrm{tt})$ or different $(\mathrm{TT}$ or $\mathrm{tt})(\mathrm{Tt})$.

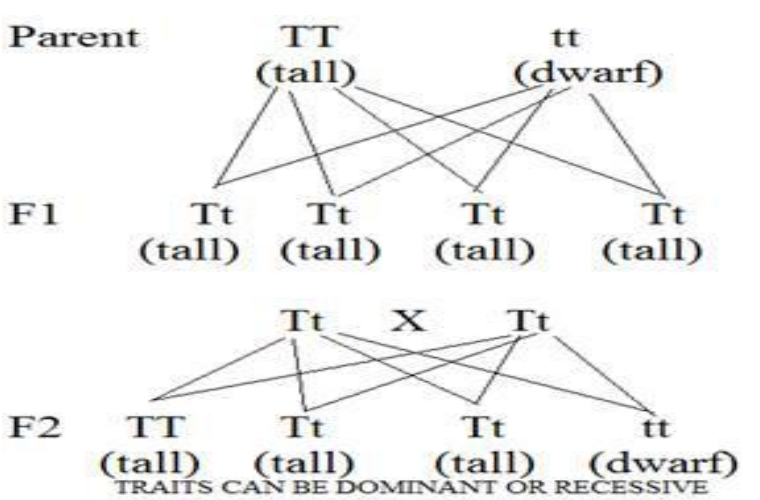

Fig 3. Dominant and Recessive alleles

The dominant allele expresses itself, while the recessive allele does not. If the dominant and recessive alleles for a specific individual are known, phenotype prediction becomes simple. Environmental factors will have a significant impact on genotype. One of several explicit functions is phenotype, which is regarded as noise and is composed of genotype $(G)$, environment $(E)$, and their interrelations $\left(G^{*} E\right)$ as shown in above Fig 3 .

Mega environments are groups of environments that share the same type of variation. The two main seasons for growing various crops are Kharif (July-October) and Rabi (March-June). We have different crops like Rice, maize, sorghum, bajra, ragi, pulses, soya bean, groundnut and cotton usually grown in kharif season where as Wheat, barley, oats, chick pea, mustard seeds are grown in Rabi season. Corn crop is considered for predicting in this paper and kharif is the season for growing corn.

Soil components, Weather components are the factors for yield prediction. Different varieties of Soil components are considered like Bulk density, Cation exchange capacity $\mathrm{pH}_{7}$, Coarse fragments, Clay, Total Nitrogen, Organic carbon density, organic carbon stock, $\mathrm{PH}$ in H20, Sand, Silt, Soil organic carbon and all of these inputs are measured with a 250 square meter resolution at six distinct depths $(0-5 \mathrm{~cm}, 5-15 \mathrm{~cm}$, $15-30 \mathrm{~cm}, 30-60 \mathrm{~cm}, 60-100 \mathrm{~cm}$ and $100-200 \mathrm{~cm})$. The different Climatic components used for estimating the yield are rainfall, radiation from the sun, Precipitation data equivalent, highest temperature conditions, lowest temperature conditions, and vapor pressure are all factors to consider. Climate components play an important role in predicting when compared with the Soil and other factors. Crop productivity, on the other hand, is nearly impossible due to a plethora of complex factors. Genotype and environmental factors, for example, frequently communicate with one another, making grain yield quite difficult. Atmospheric 
conditions, for example, often have complicated dynamic impacts that are hard to calculate accurately.

Crop productivity can be forecasted using machine learning models, Deep learning methods and artificial neural networks (Ann). Deep learning models [9] are classification learning methodologies because they have different levels of representation, each with nonlinear modules that change the representation at the present level, beginning with raw input and advancing to a progressively intuitive level. Deeper structures may also be affected by the vanishing gradient issue, that could be mitigated by using residual shortcut connections as well as numerous auxiliary heads (loss functions) for the network. To increase the accuracy of deep learning (dl) techniques, other techniques like a batch normalization, dropout, as well as stochastic gradient descent have been developed (SGD). In the literature, deep learning network techniques usually with more number of hidden layers gives good results than artificial neural network models with a single hidden layer. Deeper features, on either hand, are very complicate for training the model as well as necessitates more difficult hardware and optimization methods. Deep neural network loss functions, for instance, have a high dimensionality and are non-convex, making optimization quite difficult due to the existence of numerous local optima and saddle points.

\section{Related Work}

Soybean Flowering and Physiological Maturity Forecasted Using Neural Network Aspects [3] are founded on the hypothesis that computer simulation models of plant growth have been formulated; but the models are not always getting good results in plant developing assessment among various conditions. Fieldobserved flowering dates is being used by this study for the 'Bragg' cultivar for the purpose of scientific investigations in North Carolina, Gainesville, Quincy, Clayton and Florida. The neural network model was trained on the daily Higher and lower climatic conditions, season, and days after planting or flowering are all factors to consider. The sets of data were divided into two main categories: for training as model development as well as independent data sets for testing. The relative mean error of dataset testing for the purpose of flowering date forecasting was 0.143 days and for estimation of physiological maturity was +2.19 days. Plant continued growth estimations are useful in agricultural production because they allow the cultivators to enhance field operation planning and enhance net income as shown in below Fig 4 and Fig 5 .

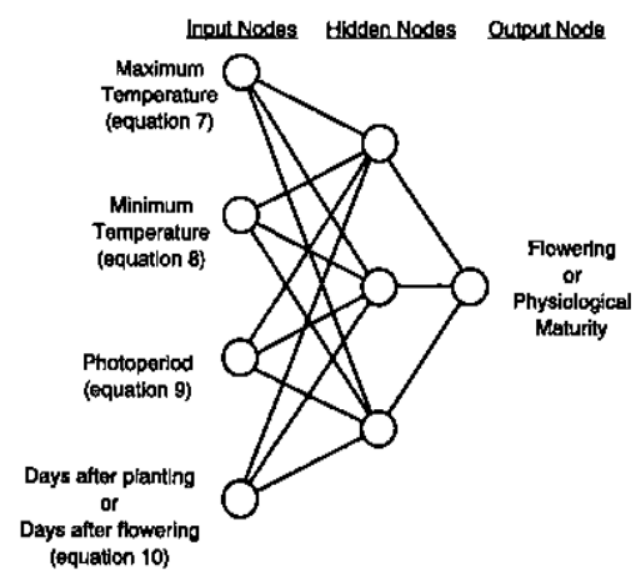

Fig 4.Inputs, connections, and structure of a neural network model are depicted using 3 distinct hidden nodes.

When seed germinates, the vegetative and reproductive development processes begin, and these processes end when the harvest matures. Temperature is a key climate variable influencing plant growth. Furthermore, duration, or the length of the daily light period, can influence increases the productivity in some species. Existing simulation models struggle to correctly estimate development in a variety of areas where either temperature or photoperiod varies. Extended preprocessing was required before feeding the data into the neural network models. The findings for the three thresholds in equations 7 through 10 were entirely dependent on pre - processing phase. It was not possible to conduct an intensive research of all threshold values and neural network requirements.

In this study, we anticipate maize yields using linear regression models and artificial neural networks models [4]. From 1962 to 2004, multiple linear regression models (MLR) and non-linear artificial neural network model (ANN) designs were used in forecasting future maize crop in China's Jilin region, with climate and fertilizer as predictor variables. Production was set to be determined by July-August rainfall, September precipitation, and fertilizer application. Fertilizer is used as a dominant forecaster in the ANN model and was found to be non-linearly related to output. For obtaining the fertilizer data of maize there is a difficulty so the studies used previous years yield tested data. Estimation skill scores derived from cross- validation technique andretroactive validation technique revealed that ANN models perform better than MLR and persistence. Due to the non-stationary nature of the data, in the analysis of forecasting skill, cross-validation method was found to be less reliable than retroactive evaluation. Researchers used two approaches to study crop productivity in relation to environmental variables: mechanistic models and empirical models. For analyzing relationships between crop, soil types, 
climatic conditions, and ecology, mechanistic approaches that depend on physiological methods for crop development are best. Because non-linear relationships identified by an ANN model are more difficult to understand than linear relationships identified by MLR, ANN has been dubbed a "black box" technique. As a result, the ANN extrapolates with greater care than a linear function. ANN gives low values compared with MLR solution and hence decides that nonlinear relationship is better. In present research, where interpretation occurred, the ANN model's bounded functional shape gave ANN an added benefit over MLR. Machine Learning Methods for Efficient Forecasting in India [5] Amount of rain, atmospheric pressure, chemical fertilizer, pesticides, ph level, and other climate patterns and parameters all have an impact on crop production. After recognizing the linear relationship among yield and these parameters, reliable yield prediction is needed.

Machine Learning techniques can improve by distinguishing and presenting the consistency and pattern of drive information, machine execution can be improved without the need for characterized computer programming. This survey used a variety of neural network models to assess crop yield on crop yield datasets from multiple areas and crops, including Linear Regression model, Gradient Boosting Regressor model, Random Forest Regressor model, Decision Tree Regressor model, Polynomial Regression model, and Ridge Regression model. Mean absolute error (MAE), mean squared error (MSE), root mean square error (RMSE), R-square, and cross validation are the measures used to compare the accuracy of these strategies. Findings indicate that the Gradient Boosting Regressor has a precision of 87.9 percent with cross validation runs.

Deep Learning techniques in Agriculture [8] is a comparatively recent, cutting-edge image processing technique and data analysis technique with best outcomes and massive results. Deep learning technique has recently made its way into agriculture after being used efficiently in other fields. We assess the sustainable agricultural challenges under consideration, as well as the models and theories employed, information sources, nature, and pre-processing as well as the improved ability achieved based on the criteria used at each work under considering. In addition, we compare deep learning to other popular technologies in terms of classification or regression efficiency differences.

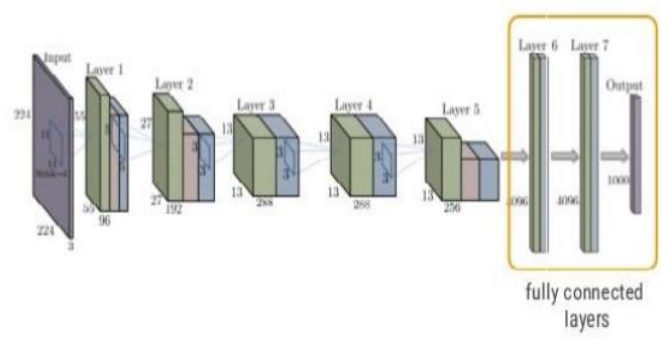

Fig 5. CaffeNet, example $\mathrm{CNN}$ architecture

Deep learning outdoes prevailing commonly used image processing method in terms of accuracy, according to our findings. Images make up a sizable portion of the information gathered through remotely sensed data. Images provide a clear understanding of farming in many cases and can be used to address a variety of issues. As an outcome, imaging analysis has become an interesting subject in agriculture, and intelligent data collection methods are used in a wide range of farming applications for image identity verification, outlier detection, and so on. DL extends traditional ML by incorporating more "depth" (complexity) to the model and transforming data from multiple capabilities that allow data representation in a systematic order, through many levels of abstraction.Based on the network architecture, DL may contain a wide range of modules. A basic problem in computer vision, not just deep learning, is that data preprocessing is frequently required and time-consuming, particularly when satellite and images are involved.The high dimensionality and slight number of training samples of hyper spectral data are two issues. Aside from that, existing datasets do not always effectively define the problem. When considering corn returns, for example, it was important to compensate in cultivation data such as fertilization and water management, external influences other than weather data can be considered. Convolutional Neural Networks (CNN) are used to capture similar spatial functioning of different features and combine them to model yield response to nutrient and grain rate management [6]. To define a new dataset for training and test sets the $\mathrm{CNN}$ model, nine on-farm corn field studies are used. When compared to multiple regression analysis, the test dataset RMSE is reduced by up to 68 percent, and by up to 29 percent when compared to a random forest. When we look at the harvest data collected within 50 meters of the field's boundaries, we can see that the input variables centered on such cells cover an area with no data.

As shown in below Fig 6 and Fig 7, Fig 8,This survey modeled yield response to nitrogen and seed rate management using five different field features (nitrogen and grain rates, elevation map, soil electro conductivity, 
and spatial data). Other risk factors and management techniques that have an effect on global field impacts gain response to site-specific planning. These parameters differ from field to field while changing progressively within the same field.

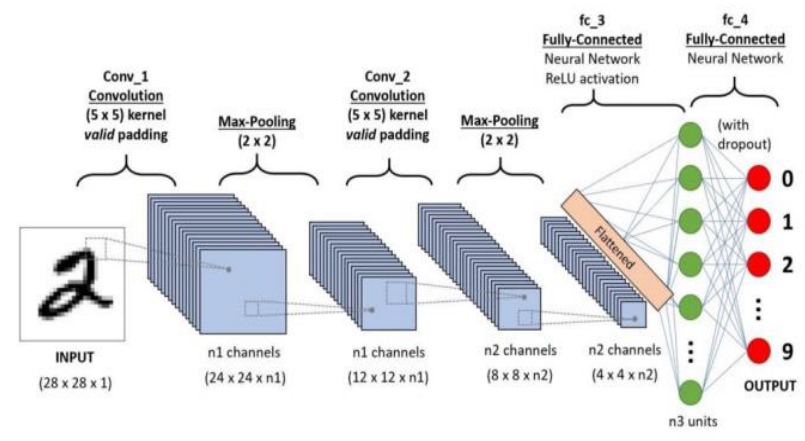

Fig 6.Convolutional Neural Network

Growing plants Date, Hybrid Maturity, and Weather Conditions Influence Corn Yield and Crop Stage [7] In Iowa, USA, we examined wheat crop and phenology data from a multi-location, year, hybrid comparative maturity, and planting date study. Our goals were to find the best combination of sowing date and comparable maturity to maximize corn seed yield per weather, as well as to mitigate the effects of using "full-season hybrids" when harvesting occurs well after the optimum planting date. According to ANOVA, $70 \%$ of the variability in seed yield was directly related to planting date and only $10 \%$ to associated to maturity, stating that short and full-season hybrid relative maturity periods generated equivalent to seed yield regardless of when they were sown as long as the farms reached maturity prior to harvesting. According to our findings, the time to grain yield is a good predictor of expected higher yield, with a critical stage (after which yield is reduced) of 23 July for Iowa. Furthermore, we discovered that keeping a lower limit growing degree of $648^{\circ} \mathrm{Cday}$ as during the grain-filling period enhanced maize yield. Conclude, the studied information will help Iowans in making sowing date decisions on the basis on hybrid relative maturity. Throughout most of the growing season (April-October), weather conditions at our sampling locations were relatively inconsistent. The Northeast place had the coolest site-year in 2014, while the Southeast place had the hottest year in 2016. In aspects of rainfall, 62 percent of the site-years were wet, 24 percent became dry, and 14 percent were indeed a mix of the two .

Maize seed yield, silking time, and other considerations, as well as grain-filling duration, are all affected by the planting date. In terms of seed yield and phenology, PD had a bigger influence than RM. Farmers in Iowa will benefit much during the growing season if they plant full-season hybrids all year; nevertheless, the real effect of RM fades over time. Immigrants came to warmer climates with a longer growing season as southern climates warm. A shortened growing season was blamed for the harvest penalty associated with delayed planting. Farmers typically select a mixture relative maturity well in order to progress of planting; however, our research shows that combination RM has a very minor impact on grain yield for any given PD when harvested before planting.

Grain yield estimation and climate making predictions using machine learning techniques for Assessment of the impacts on agriculture [9] Weather has a significant impact on crop productivity A increasing previous research models this transaction in order to estimate the threat of climate change on the industry. A yield modeling approach that makes use of a semi-parametric variant of a deep learning to account for nonlinear dynamic interactions in high-dimensional datasets, as well as known geometrical structure and unfathomable cross-sectional heterogeneity. We show that using corn yield data from the Midwest of the United States, this model outperformed both traditional statistical methods and entirely nonparametric neural networks in predicting yields of years denied during model training. We show that climate change has a major negative impact on corn prices, but it is less intense than the impacts projected using statistical models. Abatzoglou's gridded surface meteorological database (METDATA) is used to generate the meteorological data (2013). Bagging improved both the parameterization and the SNN's precision, but the bagged SNN consistently outperforms. Both OLS regression and the SNN were outpaced by the fullynonparametric neural net, which was learned in the same way as the SNN but lacked simulation terms.

Incorporating machine learning and crop simulations improves crop yield estimation in the Corn Belt of the United States [10].The goal of this research is to see if merging crop modeling and machine learning (ML) tends to improve grain yield projections in the Corn Belt of the United States. The primary objectives were to identify whether such a hybrid approach would result in better assumptions, which hybrid model mixtures provide much more better estimates, and which crop modeling attributes are most effective when combined with ML for maize crop prediction. The researchers noted that using simulation crop modeling as input features in ML models can lessen yield estimation root mean squared error (RMSE) by 7 to $20 \%$. Furthermore, we investigated the selective complicity of APSIM attributes in ML forecasting and concluded that soil moisture-related APSIM variables have the greatest impact on ML projections, followed by crop-related and weather-related APSIM variables. This finding implies that weather reports alone are inadequate, and that $\mathrm{Ml}$ 
algorithms require more surface water inputs to produce more accurate yield forecasts.

Estimation of crop prices using supervised machine learning methods [11] and our focus is primarily on agriculture. Farmers are the most important people in agriculture. Farmers face massive losses if the price falls after the cultivation. This paper proposes estimating and planning crop prices for the next 12 months. The information is provided to users via a Flask web page, which is powered by impactful machine learning techniques and innovations and has a user-friendly interface as a whole. The accumulated training datasets contain a wealth of information for forecasting market price and demand. The Decision tree Regressor supervised machine learning algorithm was used in our model. It has been honed on a number of Kharif and Rabi seasons to improve efficiency.

Estimation of Crop Production by Robots [12] Cultivation is an entirely manual endeavour. The use of machine learning methods to implement any type of automation is still in its beginning phases. The focus of this article is to present a contextual approach for introducing machine learning methods into the cropping process. A comparison of machine learning algorithms was performed in order to determine which algorithm is the most accurate in predicting the best crop for a specific plot of land. The research looks at six major food crops in Bangladesh: Aman rice, Aus rice, Boro rice, potato, wheat, and jute. Multiple Linear Regression (MLR) produced the most accurate results during the evaluation and was implemented into an Android application. The android platform system can also generate a timeline of the entire farming process for a specific crop, including when to apply fertilizer. Except for wheat and potatoes, MLR offers greater prediction in this case. KNN yields superior performance for wheat and potato. Mostly as result, the error is 0.40 percent for one and 6.26 percent for the other.

Deep Learning Methods for Yield Estimation [13]. Automatic crop monitoring and yield prediction are now possible thanks to artificial intelligence. The categories were created entirely around five networks: ANN, CNN, DNN, RNN, and combination networks. The feed forward's ANN and DNN were examined, and both offered an average predictive accuracy of 60-70 percent. The large proportion of agricultural processing was carried out using images and timely monitoring. CNN significantly outperformed both DNN and ANN models, with efficiency of 80-85 percent. The only drawback of $\mathrm{CNN}$ in this research was that its projections were based purely on training sample rather than real-time past results. A few work was aimed at improving the yield prediction even more and to avoid loss during the yield. The RNN makes use of an LSTM-LSTM combination, which allows for the addition of data storage. When particularly in comparison to the other three systems, it involves the feedback loop that $\mathrm{CNN}$ fails, evaluated to the high classification level's average estimation of $83-$ 89 percent.

\section{Proposed Work}

\subsection{Dataset Description}

For Crop yield prediction we need to select the crop here we selected the crop called Corn and going to forecast the yield based on the dataset and the applied algorithms. Dataset is the first thing we need to select here we collected the dataset from various sources like Syngenta and it mainly consists of the variables like nw, ns, np, nss where the number of weather elements is nw, and the number of soil particles is ns. measured at different depth, $n p$ is the planting component and nss is the soil component measured at the surface The six weather components considered are rainfall, radiation from the sun, snow water equivalent, highest temperature, lowest temperature, and air humidity for csv files they are named as Wij where $\mathrm{i}$ is the weather component index and consider the six weather components i.e $i=1, \ldots 6$ and $\mathrm{j}=$ index of week of year for every year there are 52 weeks so $j=1, \ldots .52$. There are 11 Soil components like bulk density(bdod), Caption exchange capacity at $\mathrm{Ph} 7(\mathrm{cec})$, Coarse fragments(cfvo), clay, Total nitrogen, Organic carbon density(ocd), Organic carbon stock(ocs), ph in H20(phh20), Sand, Silt, Soil organic carbon measured at six different depths $(0-5 \mathrm{~cm}, 5-15 \mathrm{~cm}$, $15-30 \mathrm{~cm}, 30-60 \mathrm{~cm}, 60-100 \mathrm{~cm}, 100-200 \mathrm{~cm})$ with 250 square meter resolution in csv files they can be named as $\mathrm{Sij}$ where $\mathrm{i}$ is the soil component $\mathrm{i} . \mathrm{e} \mathrm{i}=1,----11$ and $\mathrm{j}$ is the index of depth $j=1,---6$. Planting time component is considered as np in csv files they are named as pi where $i$ is the index of the planting date week $i=1,---16$. The crop performance dataset includes the identified average yield for corn between 1980 and 2019 across 1,176 countries for corn in 13 states including Indiana, Minnesota, Kansas, North Dakota, Missouri, Illinois, Iowa, Nebraska, South Dakota, Ohio, Kentucky, Michigan, and Wisconsin. Organizational data is generally the cumulative percentage of cultivated fields in each state each week starting in April of each year. Grid map approach is followed which means horizontal and vertical lines to identify locations on map.

\subsection{System Architecture}

The System Architecture of proposed work is like first consider the dataset and preprocesses the data then split into train and test data apply the classification techniques and predict the results. Here for this forecasting purpose Corn dataset is considered from 
open source challenge platform like Syngenta [2]. Cleaning, integration, reduction, and transmission are all methods of data preprocessing. We need to get rid of any data that isn't absolutely necessary. The procedure of detecting and analyzing inaccurate or incorrect records from a dataset is known as data cleaning. Real-world data frequently contains noise and missing values, and it may be in an inaccessible format that cannot be actively used for DL models. Data preprocessing is required for cleaning preparation and analysis it for various Deep Learning concepts, which increases performance and reliability. Data that is preprocessed is categorized for training and testing. Training a data means to train the model and testing is for validation process where the unseen predictions are done. The act of segregating available data into two segments, typically for crossvalidator purposes, is known as data splitting. One set of data is used to create a forecasting model, while the other is used to evaluate the performance of the model. Data separation into training and test sets is a critical step in assessing data mining models. Training percentage is taken as 80 and test percentage is 20 . When a data set is split into a training phase and a validation phase, the majority of information is used for training and only a small amount is used for test results. To train any model, no matter what type of dataset is used, the set of data must be divided into training and testing sets.In the analysis of forecasting skill, crossvalidation method was found to be less reliable than retroactive evaluation. Researchers used two approaches to study crop productivity in relation to environmental variables: mechanistic models and empirical models. For analyzing relationships between crop, soil types, climatic conditions, and ecology, mechanistic approaches that depends on physiological methods for crop development are best.

\section{Flowchart for Crop Yield Prediction}

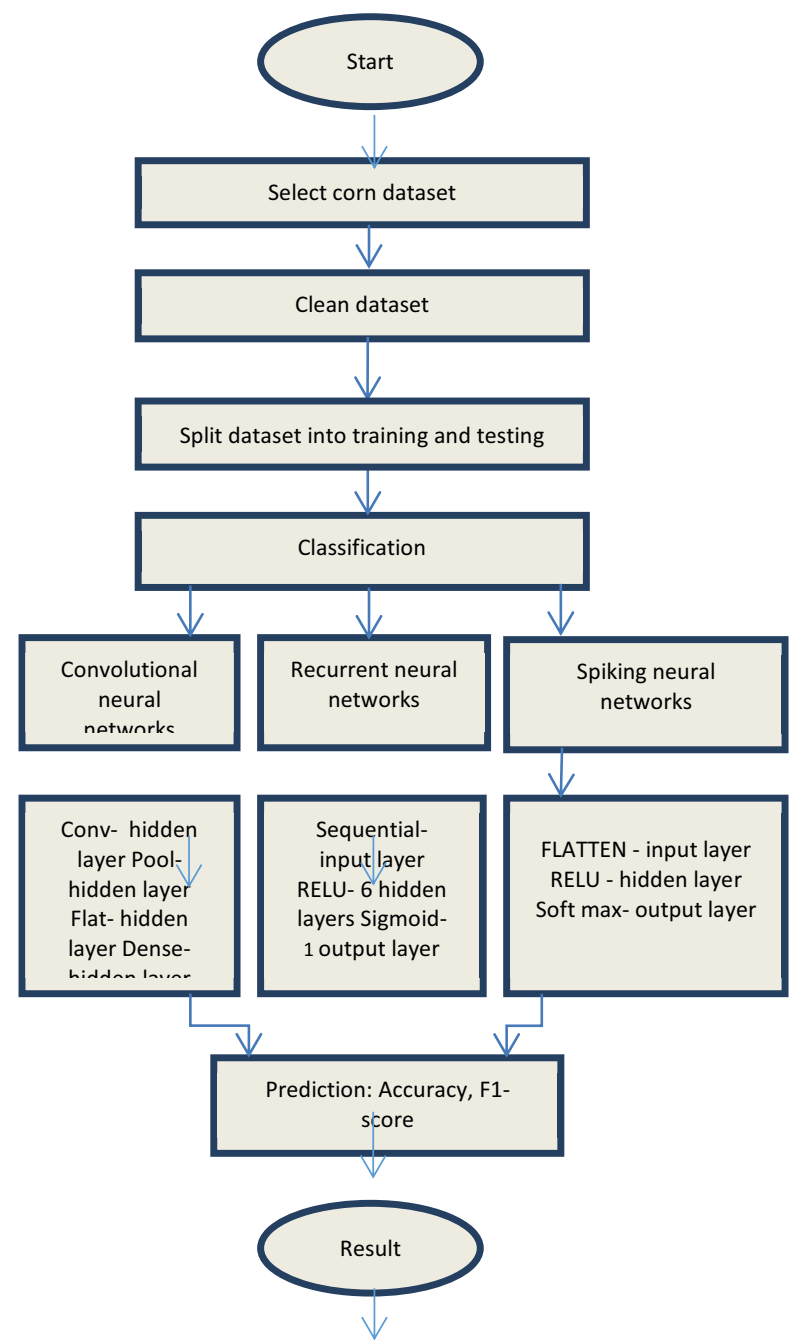

\section{Implementation}

\subsection{Deep learning Methods}

Corn yield forecasting is done using Deep Learning (DL) models. Convolutional Neural Networks (CNN), Recurrent Neural Networks (RNN), and Spiking Neural Networks are some of the deep learning models used in this paper (SNN). Neural Networks are composed of three layers: input, hidden and output layer. Input Layer: In this level various types of data is provided to the model. Number of neuron indicates the total number of features in data. Hidden Layer: The output of the Input Layer is fed into the Hidden Layer. Depending on our model and the size of the data, there could be many hidden layers. So every hidden layer may contain a different number of neurons, which is usually greater than the number of characteristics. Each layer's output is calculated by multiplying the previous layer's output by the learnable weights of that layer, followed by addition 
of transferable biases and an activation function, making the learning process non linear.

Output Layer: The hidden layer's output is then placed into a logistic function, such as sigmoid or soft max, which converts each class's output into a probability score for each class. Classification is a method of determining which of a set of characteristics a new observation belongs to, based on a learning set of data containing observations with defined category membership.

\subsection{Convolution Neural Networks (CNN)}

ConvNets also known as CNN'S consists of multiple layers mainly used for image processing and object detection. It has a number of layers that process and extract data features. $\mathrm{CNN}$ has a convolution layer with several filters that performs the convolution operation. $\mathrm{CNN}$ image categories take an image representation and process it before categorizing it. An input image is perceived by computers as an array of pixels, the size that rely on the quality of image. Depending on the resolution of an image, it will see $\mathrm{h} x \mathrm{w} \times \mathrm{d}(\mathrm{h}=$ Height, $\mathrm{w}=$ Width, $\mathrm{d}=$ Dimension). For convolution Neural Networks (CNN'S) we use four hidden layers i.e. convolution layer, Pool layer, flat layer, Dense layer. Conv1D is used and in this the kernel moves only in one direction applicable for Time series data, input and output of Conv1D is two dimensional. Convolution is a mathematical operation performed on two objects to generate an output that expresses how the shape of one is modified by the other. This calculation finds a special functionality in the input data and needs to return a result enclosing that feature's relevant data. This is known as a feature map. The process of merging is known as pooling. So it's primarily for the purpose of reducing data size. Max pooling is considered which takes the highest value within the box. One -dimensional array is considered as a method of converting input data to next layer also treated as flattening. The convolutional layer outcome is flattened to produce a single long feature vector. It is also correlated to the final classification model, forming a fully-connected layer. We attach the final layer to a single line that contains all of the pixel data. Fully connected layer is a dense layer tells that neurons of one layer communicate with next. Adaptive Moment Estimation optimization is a stochastic gradient descent model which is based on adaptive estimation of first-order as well as second-order moments. Adaptive Moment Estimation is a deep neural network trainingspecific adaptive learning rate considered as optimization algorithm. The technique makes use of the power of adaptive learning rate techniques to identify individual learning rates for each parameter. The average of the squared differences between the observed and predicted values is used to calculate the Mean Squared Error loss. The output is always positive, regardless of the sign of the predicted and actual values, and the value obtained is 0.0 .

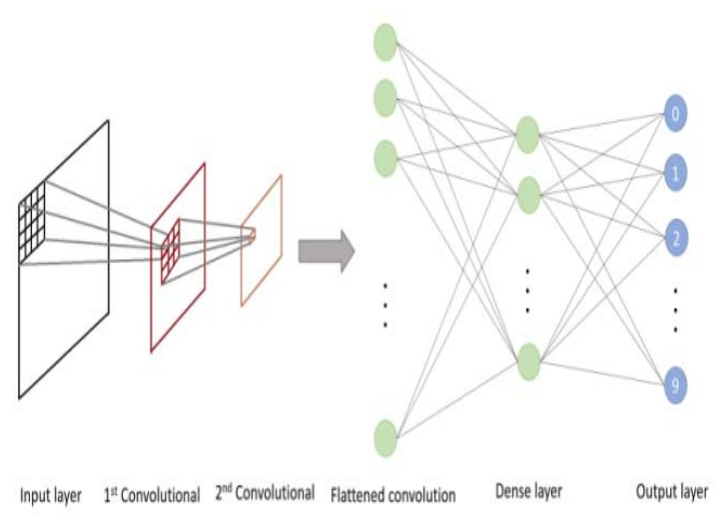

Fig 7. Convolutional Neural Networks

\subsection{Recurrent Neural Networks (RNN):}

One type of artificial neural network is recurrent neural network that can perform operations on some files like audio files, data files etc. All input and output values are independent in neural networks whereas in RNN, the hidden layers remember the data that was done in a previous layer and give that remembered data to the next layer as input so that it can perform operations on data. This process is repeated until the desired output is obtained. The rectified linear activation function, abbreviated RELU, is a piecewise linear function that retrieves the user input if it is positive and indirectly if it is negative. It has become the default activation function for few types of neural networks and it is easier to train and frequently results in better performance. Activation functions for a Rectified Linear Unit with six hidden layers. In multi-layer networks, the vanishing gradient issue restricts the use of the sigmoid and hyperbolic tangent activation functions. The rectified linear activation function solves the vanishing gradient problem, allowing features to learn more quickly and work better. When building multilayer Perceptron and 
convolutional neural network models, the rectified linear activation act as standard activation. Input layer is sequential and output layer is sigmoid.

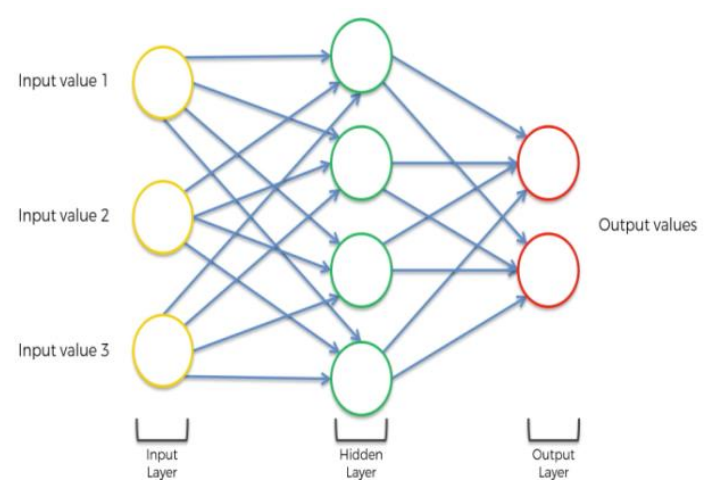

Fig8. Recurrent Neural Networks

\subsection{Spiking Neural Networks (SNN):}

Spiking neural networks (SNNs) were artificial neural networks that resembled natural neural networks. SNNs incorporate time into their operating model in terms of neuronal and synaptogenesis state. According to the principle, the neurons in the SNN do not need to transmit data during every propagation cycle. Information is only sent when a membrane potential - an inherent property of the neuron associated to its electrical charge on the membrane - reaches a specified value known as the threshold. When the membrane potential reaches a certain threshold, the neuron fires and transmits the signal to other neurons, which modify their potentials in reactions to the signal. The input layer is flattening. Flattening is the process of converting data into a onedimensional array for input into the next layer. We flatten the layer output to create a unified long feature vector. It is also linked to the last classification model, resulting in a fully connected layer. The ReLU layer is the hidden layer. The rectified linear activation function (ReLU) is a piecewise linear function that, if the input is positive, effectively generates the input; otherwise, it outputs zero. The rectified linear activation function solves the vanishing gradient problem, allowing models to learn more quickly and function better. The output layer, the Softmax layer, broadens this concept into a multi-class work environment. The total of those decimal likelihoods must equal 1.0. The number of neurons in the Softmax layer must be the same as number of neurons in the output layer as shown in below Fig 9.

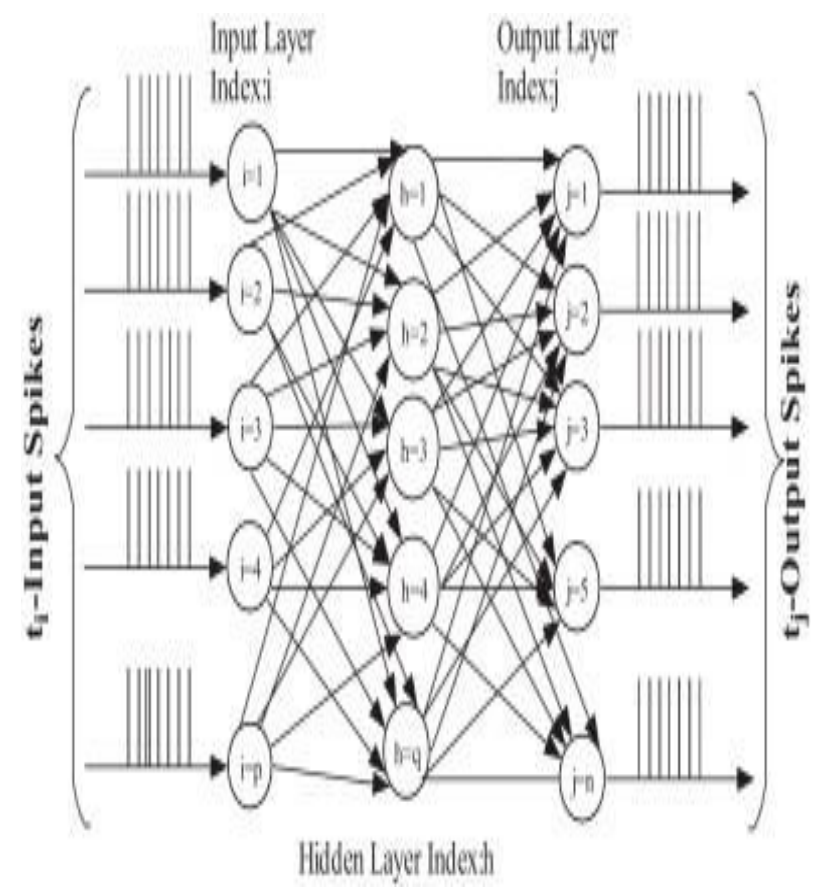

Fig 9.Spiking Neural Networks

\section{Results}

Tensor flow, an open-source software library, was used to create the three deep neural networks in Python. The training phase for each neural network took about 1.4 hours on a Tesla K20m GPU. For comparative analysis, we used three other well-known forecasting models: Convolutional Neural Network models (CNN), Recurrent Neural Network models (RNN), and Spiking Neural Networks models (SNN). To ensure better comparisons, we wrote all of these modeling techniques in Python and evaluated them in the same software and hardware environments. The overall classification and prediction will be used to generate the Final Result. The effectiveness of the proposed approach is assessed using metrics such as, the ability of a classifier is referred to as its accuracy Class label is predicted correctly and the accuracy tells how good a given predictor can guess the value of attribute for new data.

$$
\mathrm{AC}=\frac{T P+T N}{T P+T N+F P+F N}
$$

$$
\mathrm{F}-\text { measure }=\frac{2 T P}{2 T P+F P+F N}
$$




\subsection{Results for Convolutional Neural Network}

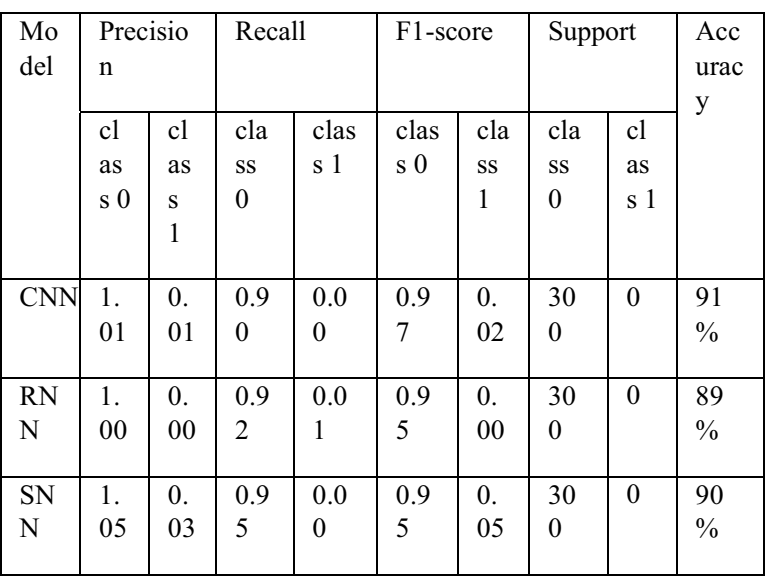

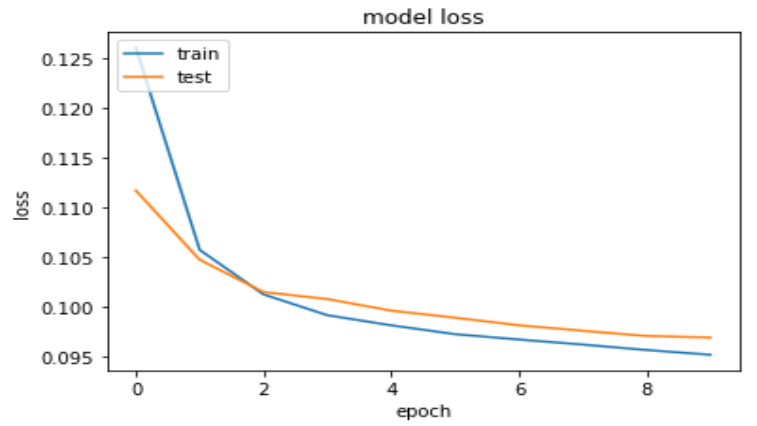

\subsection{Results for Spiking Neural Network}

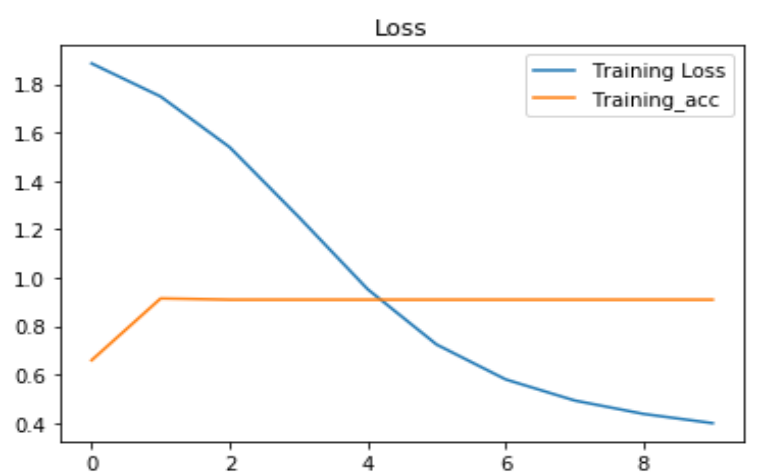

\subsection{Results for Recurrent Neural Network}

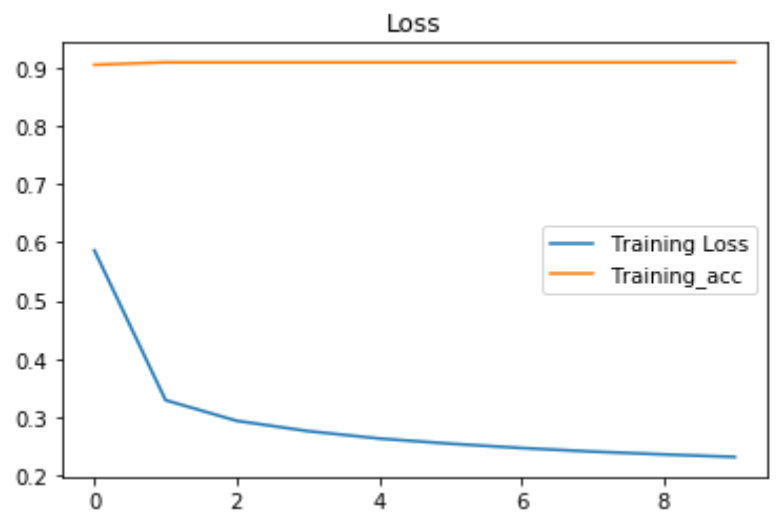

\subsection{Result Analysis}

The prediction accuracy of crop yield is compared with 3 models and the results are depicted in Table1.

Table 1: Comparing different measures for CNN, RNN, SNN

\section{Conclusion}

We described a deep learning-based framework for forecasting climate information and management in this study practices that successfully predicted corn yields across the entire Corn Belt in the United States. Most importantly, our approach went beyond estimation by providing major findings that aided in the explanation of yield estimation (variable importance by time period). The method developed are convolutional neural networks (CNN) and Spiking neural networks (SNN). The CNN component of the framework was designed to capture the intrinsic temporal relationship of weather data as well as the spatial interconnections of soil data measured at different depths underground. The model's RNN component was designed to capture the rising trend in crop yield over time as part of ongoing improvements in plant reproduction and management practises. SNNs work with spikes, which are discrete events that happen at specific points in time, instead of continuous values. The occurrence of a spike is determined by nonlinear equations that depict various biological processes, the most significant of which is the membrane potential of a neuron. When a neuron attains 
a certain potential, it spikes, and the potential of the neuron is reset. Several variables, including climatic conditions, soil, and management, had a large impact on the model's effectiveness. In unknown environments, the suggested framework accurately predicted yields, and it could thus be used in tasks requiring long-term yield forecasting. One of the most difficult aspects of deep learning techniques is their black box nature. To make the current proposal less of a black box and more concise, feature selection was back propagation method that is used for trained CNN-RNN model. The feature selection model estimated the individual impact of weather components, soil type, and management variables, as well as the time span when these factors get to be significant, and that is a study development. This method could be used to solve other research issues. Similarly, a hybrid could be classified as low-yielding or high-yielding depends on the performance in comparison to other varieties in the same location.

\section{References}

1. Khaki, Saeed\& Wang, Lizhi. (2019). Crop Yield Prediction Using Deep Neural Networks.Frontiers in Plant Science.10.

10.3389/fpls.2019.00621.

2. Syngenta (2021). Syngenta Crop Challenge In Analytics. Available online at: https://www.ideaconnection.com/syngenta-cropchallenge/challenge.php/

3. Elizondo, David \& McClendon, R.W.. (1994). Neural Network Models for Predicting Flowering and Physiological Maturity of
Soybean. Transactions of the American Society of Agricultural Engineers. 37. 981-988. 10.13031/2013.28168.

4. Matsumura, K. \&Gaitan, Carlos \& Sugimoto, K. \& Cannon, Alex \& Hsieh, William. (2015). Maize yield forecasting by linear regression and artificial neural networks in Jilin, China.1-12.

5.PayalGulati, Suman Kumar Jha, 2020, Efficient Crop Yield Prediction in India using Machine Learning Techniques, INTERNATIONAL JOURNAL OF ENGINEERING RESEARCH \& TECHNOLOGY (IJERT) ENCADEMS - 2020 (Volume 8 - Issue 10),

6. Barbosa, Alexandre\&Trevisan, Rodrigo \&Hovakimyan, Naira \& Martin, Nicolas. (2020). Modeling yield response to crop management using convolutional neural networks.Computers and Electronics in Agriculture. 170. 105197. 10.1016/j.compag.2019.105197.

7. Baum, Mitch \&Archontoulis, S. \&Licht, Mark. (2018). Planting Date, Hybrid Maturity, and Weather Effects on Maize Yield and Crop Stage.Agronomy Journal.111. 10.2134/agronj2018.04.0297.

8.AndreasKamilaris, Francesc X. Prenafeta-Boldú,Deep learning in agriculture: A survey,Computers and Electronics in Agriculture, https://doi.org/10.1016/j.compag.2018.02.016.

(https://www.sciencedirect.com/science/article/pii/S01681699173088 03)

9. Khaki, Saeed\& Wang, Lizhi\&Archontoulis, Sotirios. (2019). A CNN-RNN Framework for Crop Yield Prediction.

10. Shahhosseini, Mohsen \& Hu, Guiping\& Huber, Isaiah \&Archontoulis, Sotirios. (2021). Coupling machine learning and crop modeling improves crop yield prediction in the US Corn Belt. Scientific Reports. 11. 10.1038/s41598-020-80820-1.

11. Mulla, Sadiq\&Quadri, S..(2020). Crop-yield and Price Forecasting using Machine Learning.TheInternational journal of analytical and experimental modal analysis. XII. 1731-1737.

12. T. Siddique, D. Barua, Z. Ferdous and A. Chakrabarty, "Automated farming prediction," 2017 Intelligent Systems Conference (IntelliSys), 2017, pp. 757-763, doi: 10.1109/IntelliSys.2017.8324214.

13. Dharani, M \& Thamilselvan, R \&Natesan, P \&Kalaivaani, PCD \&Santhoshkumar, S. (2021). Review on Crop Prediction Using Deep Learning Techniques. Journal of Physics: Conference Series. 1767. 012026. 10.1088/1742-6596/1767/1/012026. 